\title{
REUSE OF SOFTWARE ASSETS FOR THE NASA EARTH SCIENCE DECADAL SURVEY MISSIONS
}

\author{
Chris A. Mattmann ${ }^{1,4}$, Robert R. Downs ${ }^{2}$, James J. Marshall ${ }^{3}$, Neal F. Most ${ }^{3}$, Shahin Samadi ${ }^{3}$ \\ ${ }^{1}$ Jet Propulsion Laboratory \\ California Institute of Technology \\ Pasadena, CA 91109, USA \\ mattmann@jpl.nasa.gov \\ ${ }^{3}$ INNOVIM \\ NASA Goddard Space Flight Center \\ Greenbelt, MD 20771, USA \\ James.J.Marshall@nasa.gov \\ ${ }^{2}$ Center for International Earth Science Information \\ Network \\ Columbia University \\ Palisades, NY 10964, USA \\ rdowns@ciesin.columbia.edu \\ ${ }^{4}$ Computer Science Department \\ University of Southern California \\ Los Angeles, CA 90089, USA \\ mattmann@usc.edu
}

\begin{abstract}
Software assets from existing Earth science missions can be reused for the new decadal survey missions that are being planned by NASA in response to the 2007 Earth Science National Research Council (NRC) Study. The new missions will require the development of software to curate, process, and disseminate the data to science users of interest and to the broader NASA mission community. In this paper, we discuss new tools and a blossoming community that are being developed by the Earth Science Data System (ESDS) Software Reuse Working Group (SRWG) to improve capabilities for reusing NASA software assets.
\end{abstract}

Index Terms - ESDS, Software Reuse, Reference Architecture.

\section{INTRODUCTION}

The future Earth science missions at the National Aeronautics and Space Administration (NASA) promise to provide an explosion of data and a platform for science that previously was unachievable using existing hardware, software, and assets. Instrument resolution is increasing, as is the ability of software and hardware to deal with data volumes that will easily grow to the $10-100$ petabyte range in the next five years [1]. Over the past twenty years, NASA has invested in software to support all phases of the Earth science mission pipeline. These investments include components and architectures that support science data processing at Science Investigator-led Processing Systems (SIPS), data archival and dissemination at the Distributed Active Archive Centers (DAACs), and ad-hoc data analyses and custom product generation using DAAC-provided data [2]. This general flow is shown in Figure 1.
For example, the Moderate Resolution Imaging Spectroradiometer (MODIS) Data Processing System (MODAPS) has evolved over time to support higher data processing rates and the production of data products for additional Earth-observing instruments by enhancing its architecture [3]. In addition, several recent efforts [4] to standardize process management and control for both the Orbiting Carbon Observatory (OCO) missions, as well as the NPOESS Preparatory Project (NPP) joint NASANOAA-DOD missions, have also demonstrated the utility in the reuse of software assets.

However, to date the aforementioned efforts are the exception and not the norm. Many Earth science data system components and architectural patterns are reconstructed for each mission. There are a number of reasons for this including: (1) the distributed scientific expertise of NASA, (2) the desire to have that expertise co-located with the data as it is processed and delivered for wide dissemination, (3) procurement practices, where contract and equipment resources are stove-piped into separate contracts and programs, and (4) each scientific community purports a unique set of requirements for data processing and data products, that may not easily lend itself to justify reuse.

The paradigm of NASA missions is changing, primarily due to the upcoming missions identified in the National Research Council's Earth Science and Applications from Space decadal survey [5] (as well as other future "decadallike" missions). It is even more imperative that NASA look to reduce costs, increase software productivity, explore areas for consolidation of homogeneous services, and ultimately promote and facilitate a culture of reusing successful software assets and patterns across its missions.

Software reuse can help inform the successful design of future NASA missions in a number of different ways, in particular through: (1) identification and selection of 


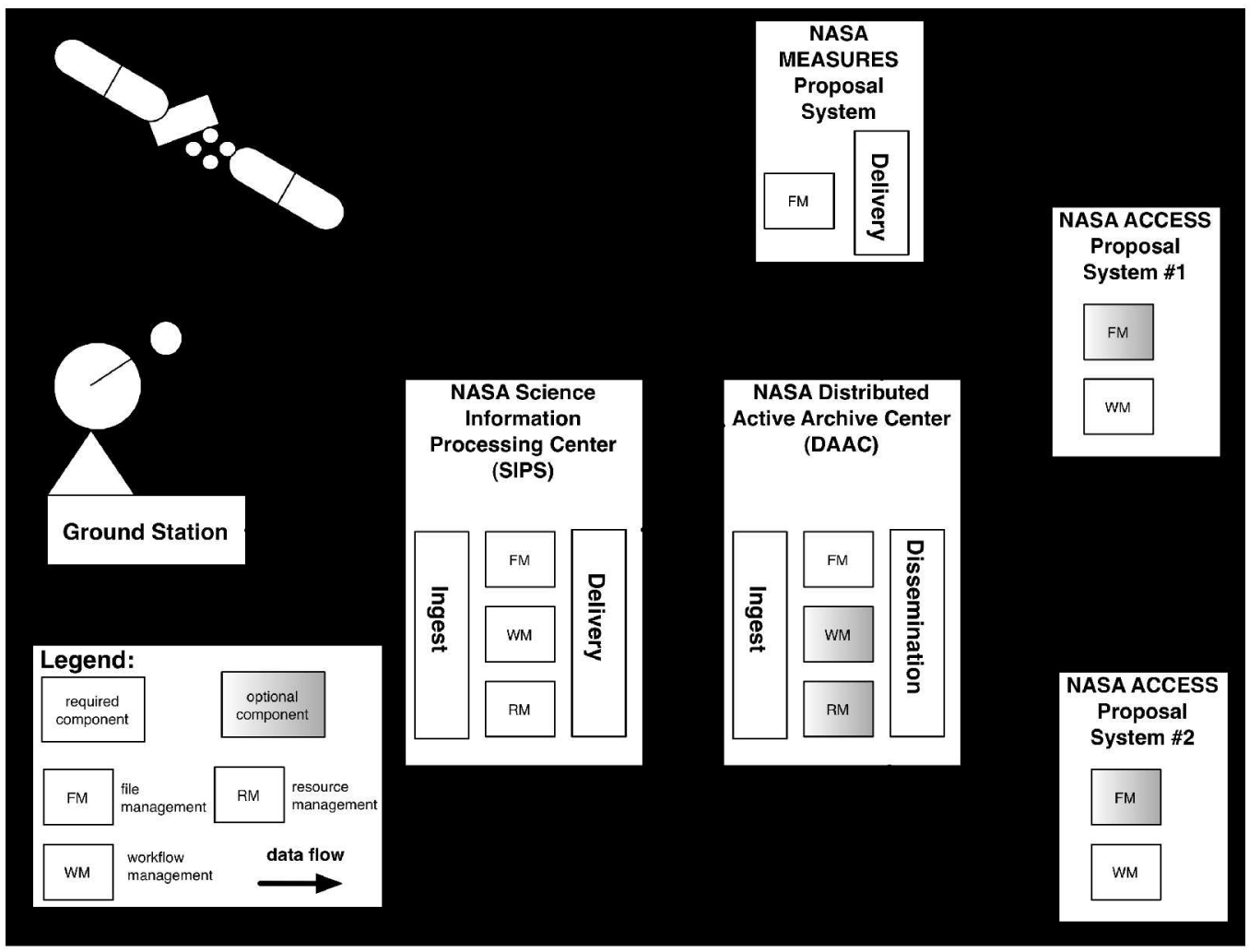

Figure 1. The NASA Earth Science Context. Data is taken by instruments on spacecraft and sent to ground stations, which move the data to SIPS. DAACs are responsible for long-term archiving of the information, and dissemination. Ad-hoc analyses occur in the ACCESS and MEaSUREs programs.

existing, proven Earth science software components (or software components applicable in Earth science data systems) whose reuse saves development costs and time; (2) application of existing architectural styles and patterns [6] that induce specific quality attributes (reliability, scalability, etc.) in the resultant software; and (3) identification of new assets developed for missions which are of broader applicability, and themselves should be disseminated to the community, to name a few.

Reusable software artifacts are not limited to just code. These assets may include algorithms and models, architectures and design patterns, systems modules and scripts, technical documentation and test results, and use metrics as well as other artifacts produced during the software development life cycle.

The NASA Earth Science Data Systems (ESDS) Software Reuse Working Group (SRWG) is chartered with the investigation, production, and dissemination of information related to the reuse of NASA Earth science software assets. One major current objective is to engage the NASA decadal missions in areas relevant to software reuse.

In this paper we will report on the current status of these activities. We will first provide some background on the SRWG in general and then discuss the group's flagship recommendation, the NASA Reuse Readiness Levels (RRLs). We continue by describing areas wherein which mission software may be reused in the context of NASA decadal missions. We conclude the paper with pointers to future directions.

\section{WORKING GROUP BACKGROUND}

The NASA Earth Science Data Systems (ESDS) Software Reuse Working Group [7] is chartered with the promotion and identification of software assets targeted for reuse in NASA's Earth Science Data System pipeline. The group is focused on architectures and technologies that facilitate software reuse. In particular, we are currently investigating software components and architectures developed to enable cloud and grid computing capabilities, as well as cyberinfrastructure for using mission and scientific data.

The flagship product of the group to date is a focused set of NASA Reuse Readiness Levels (RRLs), which have been released and are now available for use [8]. The RRLs, similar to the NASA Technology Readiness Levels (TRLs) for technology, are a nine-level guide that can be used to rank and compute the reusability of a software asset [9].

Besides the RRLs, the Software Reuse Working Group (SRWG) is also working on the development of case study documents describing efforts to leverage the RRLs in the assessment of two areas of NASA mission software: (1) the methodology and suitability of existing NASA software 
assets for inclusion in a mission; and (2) the identification, curation, and dissemination of software assets that are being developed as part of a NASA mission that can be later included. In addition, the SRWG is working on a recommendation for the packaging and distribution of reusable software assets, covering an information model for software packaging, and a classification/comparison of the state of the art in software packaging technologies.

Both of the aforementioned documents are considered a works-in-progress, and both of the documents include input from current NASA decadal missions including the Soil Moisture Active Passive (SMAP) mission and the ICESAT2 mission. We also are actively working with other tier-1 NASA decadal missions including DESDynI to best determine how and where reusable software assets could be leveraged. In the next section we will provide greater detail about the NASA Reuse Readiness Levels (RRLs) and their applicability to NASA decadal missions.

\section{REUSE READINESS LEVELS}

The NASA Reuse Readiness Levels (RRLs) have been developed for use as a measure to evaluate the potential reusability of software. The RRLs can be used to assess software that is being developed or to assess software assets that are being considered for adoption. Software can be evaluated by either using the RRLs in a simple manner to obtain a rough assessment of the software or by using the RRLs more extensively to obtain a precise assessment, which would include an assessment of the software in terms of nine topic areas.

Using the RRLs in a simple manner, the software under evaluation is compared to the brief summary descriptions of the RRLs to determine a value, from 1 to 9 , that reflects the level of the potential reusability of the software. The RRLs can be used in this way to attain a quick assessment, which lacks precision, but may be appropriate for attaining efficient assessments when comparing many competing software candidates or when only a rough estimate of the potential reusability of a software product is required.

Alternatively, extensive use of the RRLs can be applied by using a 9x9 grid to evaluate the software against each of the topic areas to determine the level of maturity that the software has attained for each of the nine topic areas. Using the RRLs in this extensive manner can be more time consuming, but enables assessment of each topic area to identify areas where additional development may be required to meet the needs of a particular software project. Prior to using the RRLs in this manner, the software requirements of the project should be identified for each topic area so that the level of effort necessary to improve the software to an acceptable level for each topic area can be determined.

A calculator is being developed for use with the RRLs. Using the RRL calculator, weights can be established for each topic area, depending on the importance of a particular topic area to meet the requirements of a particular software project, and an average overall RRL value can be calculated from assessments of topic area levels. A more advanced version of the RRL calculator, which may offer more features and/or guidance on assessing software assets, is under consideration.

Tools such as the RRL calculator enable a structured evaluation of the reusable asset as software producers and consumers measure applicability and compatibility for their particular project. We are exploring the integration of the RRL calculator with our Reuse Enablement System (RES) [10], a software portal used to track and disseminate information about reusable software assets. The RES system is currently being deploymed in the Soil Moisture Active Passive (SMAP) as a proof of concept, shown in Figure 2. In the following section, we will describe the relationship of RRLs, and associated software reuse tools to that of the NASA decadal missions.

\section{REUSE OF MISSION SOFTWARE}

The reuse of software offers opportunities for the new decadal survey missions and future space missions to reduce costs and improve the quality of the software that is either produced or used from previous efforts. Likewise, software reuse offers opportunities to obtain similar benefits when processing and re-processing data obtained from such missions. Recipients of the NASA ESDS Software Reuse Working Group Peer-Recognition Software Reuse Award [11] have demonstrated the contribution of new reusable assets and the utilization of existing reusable assets in systems development for NASA missions; for example, the National Polar-orbiting Operational Environmental Satellite System Preparatory Project's Science Data Segment reused a variety of system components to reduce development effort and help ensure reliability [12], as did the Orbiting Carbon Observatory's (OCO) Ground Data System [4].

Procedures and templates also are being developed to use the RRLs to assess the current state of readiness when assessing software from previous missions for potential

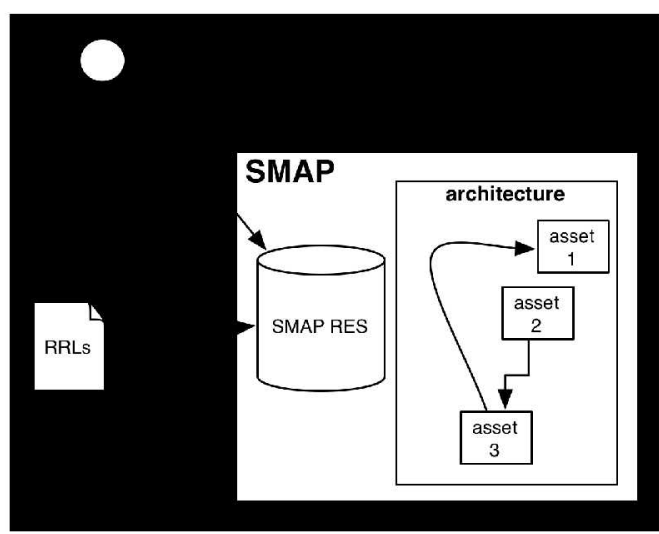

Figure 2. Using the SRWG RRLs and RES to design and implement NASA decadal missions. 
reuse in future missions. Using such tools can help to improve the usability of software created during previous missions. Such tools also can be used to assess the potential reusability of software that is being developed for new missions to improve its potential for reusability in other future missions.

In addition, using tools, such as calculators, templates, and procedures, in conjunction with the RRLs, to assess the reusability of software, can identify aspects of the RRLs that may be considered for possible improvement. Likewise, testing the use of such tools for assessing the potential reuse of software also will contribute to their refinement and inspire the development of additional tools for assessing reusability $[13,14,15]$ and can foster the consumer's confidence that the asset has been assessed as to its level of robustness and readiness for operational use.

\section{SUMMARY}

Considering the data processing needs of the new decadal survey missions, the reuse of software from previous missions offers an opportunity to leverage the investments from previous missions. The RRLs have been developed by the ESDS SRWG to assess the readiness of software for potential reuse. Using the RRLs in conjunction with other tools, such as the RRL calculator, templates, procedures, and lessons learned, can improve capabilities for reusing software in new missions and for realizing the benefits of software reuse.

\section{ACKNOWLEDGMENTS}

The authors are grateful to the members of the National Aeronautics and Space Administration (NASA) Earth Science Data Systems Software Reuse Working Group who have contributed to the efforts described in this work. Support was provided for Robert Downs under NASA contract NNG08HZ11C. This effort was supported in part by the Jet Propulsion Laboratory, managed by the California Institute of Technology, under a contract with the National Aeronautics and Space Administration.

\section{REFERENCES}

[1] R. Yang and M. Kafatos, "Massive data sets issues in earth observing," in Handbook of Massive Data Sets, J. Abello, P.M. Pardalos, and M.G. Resende, Eds. Kluwer Academic Publishers, Norwell, MA, pp. 1093-1140, 2002.

[2] H.K. Ramapriyan, J. Behnke, E. Sofinowski, D. Lowe, and M.A. Esfandiari, "Evolution of the Earth Observing System (EOS) Data and Information System (EOSDIS)," in Standard-Based Data and Information Systems for Earth Observation, Springer-Verlag, Berlin, pp. 63-92, 2010.

[3] E. Masuoka, C. Tilmes, N. Devine, G. Ye, and M. Tilmes, "Evolution of the MODIS science data processing system," Geoscience and Remote Sensing Symposium, 2001. IGARSS '01. IEEE 2001 International, vol. 3, pp. 1454-1457, 9-13 July 2001.
[4] C. Mattmann, D. Freeborn, D. Crichton, B. Foster, A. Hart, D. Woollard, S. Hardman, P. Ramirez, S. Kelly, A. Y. Chang, C. E. Miller. A Reusable Process Control System Framework for the Orbiting Carbon Observatory and NPP Sounder PEATE missions. In Proceedings of the 3rd IEEE Intl' Conference on Space Mission Challenges for Information Technology (SMC-IT 2009), pp. 165172, July $19-23,2009$.

[5] National Research Council, Earth Science and Applications from Space: National Imperatives for the Next Decade and Beyond, National Academies Press, Washington, 2007.

[6] R.N. Taylor, N. Medvidovic, and E.M. Dashofy, Software Architecture: Foundations, Theory, and Practice, John Wiley \& Sons, USA, 2009.

[7] J.J. Marshall, R.R. Downs, S. Samadi, N.S. Gerard, and R.E. Wolfe, "Software reuse to support earth science", Journal of Frontiers of Computer Science and Technology, vol. 2, no. 3, pp. 296-310, May 2008.

[8] NASA Earth Science Data Systems Software Reuse Working Group, "Reuse Readiness Levels (RRLs), Version 1.0", Available: http://www.esdswg.org/softwarereuse/Resources/rrls/, April 30, 2010.

[9] J.J. Marshall and R.R. Downs, "Reuse Readiness Levels as a Measure of Software Reusability," Geoscience and Remote Sensing Symposium, 2008. IGARSS 2008. IEEE International, vol. 3, pp. III-1414-III-1417, 7-11 July 2008.

[10] J. J. Marshall, R. Downs, C. Mattmann. Progress Towards a NASA Earth Science Reuse Enablement System (RES). To appear in Proceedings of the 11th IEEE International Conference on Information Reuse and Integration (IRI 2010), Las Vegas, NV, August 4-6, 2010.

[11] NASA Earth Science Data Systems Software Reuse Working Group, "Peer-Recognition Software Reuse Award Recipients," Available:

http://www.esdswg.com/softwarereuse/Resources/awards/reuseaward-recipients/, June 28, 2010.

[12] S. Samadi, R. Gerard, M. Hunter, J.J. Marshall, R.J. Schweiss, R.E. Wolfe, E.J. Masuoka, "Reusing Software to Build Data Processing Systems: NPP Science Data Segment Case Study," Aerospace Conference, 2007 IEEE, pp.1-12, 3-10 March 2007.

[13] J.J. Marshall, R.R. Downs, and S. Samadi, "Relevance of Software Reuse in Building Advanced Scientific Data Processing Systems," Earth Science Informatics, vol. 3, no. 1, pp. 95-100, June 2010.

[14] J.J. Marshall, R.R. Downs, and S. Samadi, "Building the Next Generation of Aerospace Data Processing Systems by Reusing Existing Software Components," in Aerospace Technologies Advancements, T.T. Arif, Ed. IN-TECH, Croatia, pp. 25-36, 2010.

[15] R.R. Downs and J.J. Marshall, "A Proposal on Using Reuse Readiness Levels to Measure Software Reusability," Data Science Journal, submitted, 2010. 